\title{
A case of a traumatic chyle leak following an acute thoracic spine injury: successful resolution with strict dietary manipulation
}

\author{
Andrea M Pakula ${ }^{1 *}$, Wendy Phillips ${ }^{2 \dagger}$ and Ruby A Skinner ${ }^{1+}$
}

\begin{abstract}
Background: Chylothorax is a rare form of pleural effusion that can be associated with both traumatic and nontraumatic causes. Thoracic duct ligation is often the treatment of choice in postsurgical patients; however the optimal treatment of this disease process after traumatic injury remains unclear [1]. We present a rare case of a thoracic duct injury secondary to a blunt thoracic spine fracture and subluxation which was successfully treated non-operatively.

Case Presentation: A 51 year old male presented as a tier one trauma code due to an automobile versus bicycle collision. His examination and radiographic work-up revealed fractures and a subluxation at the third and fourth thoracic spine levels resulting in paraplegia. He also sustained bilateral hemothoraces secondary to multiple rib fractures. Drainage of the left hemothorax led to the diagnosis of a traumatic chylothorax. The thoracic spine fractures were addressed with surgical stabilization and the chylothorax was successfully treated with drainage and dietary manipulation.
\end{abstract}

Conclusions: This unusual and complex blunt thoracic duct injury required a multidisciplinary approach. Although the spine injury required surgical fixation, successful resolution of the chyle leak was achieved without surgical intervention.

\section{Introduction}

The majority of reported cases of chylothorax are due to malignancy (50\%) specifically non-Hodgkin's lymphoma. Chylothorax due to traumatic thoracic injuries including iatrogenic post surgical injuries comprise approximately twenty-five percent of cases. Other iatrogenic complications primarily related to central access catheters make up the remaining twenty-five percent [2,3]. This disease process, if not properly recognized and treated can lead to profound respiratory, nutritional and immunological dysfunction resulting in significant patient morbidity and mortality. The available treatment modalities include conservative management with drainage and strict dietary regulation or more invasive approaches namely thoracic duct ligation $[4,5]$.

\footnotetext{
* Correspondence: APakula333@aol.com

† Contributed equally

'Department of Surgery, Division of Trauma and Surgical Critical Care, Kern Medical Center, Bakersfield, California

Full list of author information is available at the end of the article
}

\section{Case Presentation}

The patient is a 51 year old male who was struck by an automobile at 35 miles per hour while riding a bicycle. There was loss of consciousness in the field and he arrived to our level II trauma center in full spine precautions, as a tier one trauma code. His primary survey was intact and his initial vital signs were; BP 115/80, HR 84, RR 30 , O2 saturation $89 \%$ on room air which improved to $98 \%$ on a non-rebreather mask at $100 \%$. Pertinent findings on secondary survey revealed bilateral chest wall tenderness to palpation, diminished breath sounds bilaterally, upper thoracic spine tenderness to palpation, a complete loss of motor function in his lower extremities, a loss of sensory function below the level of T4 and a Glascow Coma Scale (GCS) of 15. His American Spine Injury Association Motor Score was 50. He also had a loss of his cremasteric reflex, and bulbar cavernous reflex, and had no sacral tone.

While observing strict spine precautions, the patient had chest and pelvis x-rays taken, and was then

\section{Biomed Central}


transported for computed tomography scans of the head, cervical spine and torso. Positive findings from these studies revealed multiple bilateral rib fractures with associated hemothoraces (Figure 1). He also sustained fractures and subluxation at the third and fourth thoracic levels (Figure 2). The patient was started on spinal dose steroids and strict spine precautions were maintained for anticipated surgical stabilization. Bilateral chest tube thoracostomies were placed for the hemothoraces and a arterial blood gas was then obtained which documented adequate oxygenation and ventilation given this patient's significant pulmonary injury; (pH $7.33 \mathrm{pCO}_{2} 42 \mathrm{PaO} 291 \mathrm{HCO} 321, \mathrm{O}_{2}$ saturation 97 BD-4, 2 liters nasal cannula).

The initial drainage from the left chest tube was 500 milliliters $(\mathrm{ml})$ of blood and on his second hospital day it was noted that the chest tube output was $400 \mathrm{ml}$ of milky white fluid suspicious for chyle. Biochemical analysis of the pleural fluid revealed triglycerides of 287 milligrams/decilitre $(\mathrm{mg} / \mathrm{dL})$, total protein of $2600 \mathrm{mg} /$ $\mathrm{dL}$, and LDH of 2823 units/L. These results confirmed a diagnosis of chylothorax.

Due to the complexity of the case, a multidisciplinary team approach was taken to develop the appropriate treatment regimen for this patient. The decision to attempt treatment of the chyle leak with dietary manipulation was agreed upon and the patient was started on a very-low-fat oral diet consisting of mainly fresh fruits, vegetables and whole grains. The patient was also given a semi-elemental formula, Peptamen AF, 1 can with each meal which provided additional kilocalories, protein, and medium chain triglyceride (MCT) oil in order to facilitate wound healing. Two scoops of protein powder (beneprotein) were added to each meal as well. The patient was also started on octreotide, $200 \mathrm{mcg}$ subcutaneous every 8 hours to aid in the reduction of lymph production.

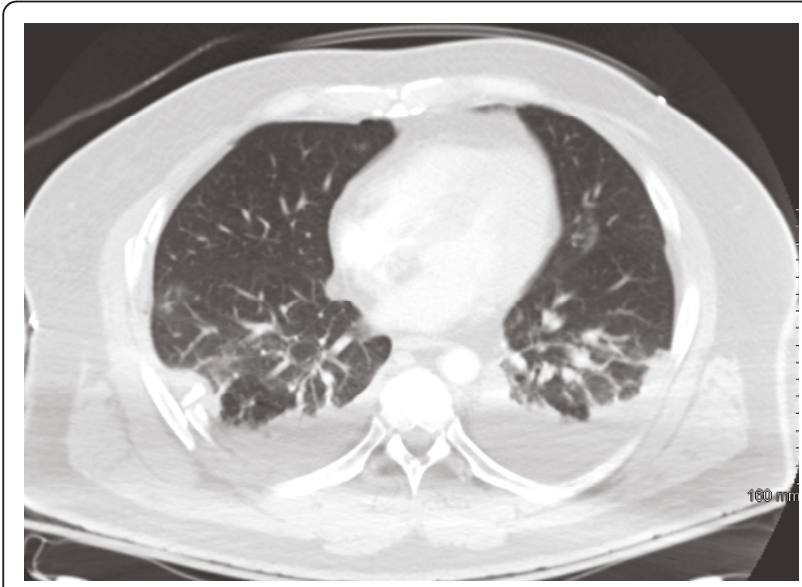

Figure $1 \mathrm{CT}$ scan of the chest illustrates bilateral pleural effusions.

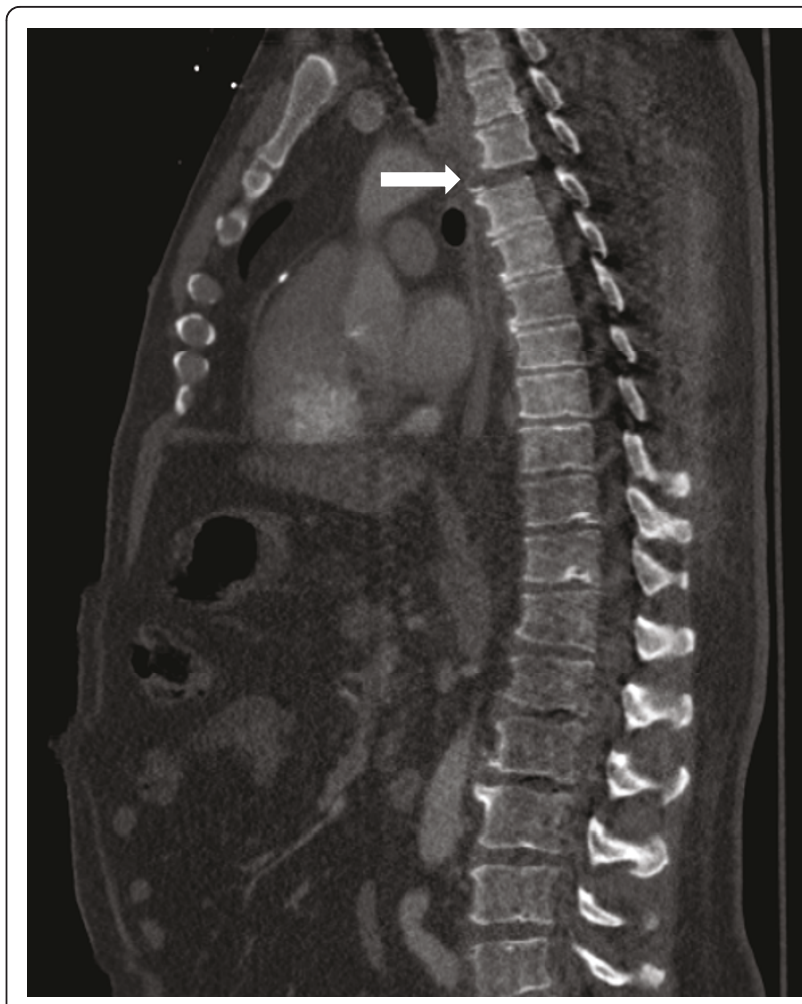

Figure 2 Lateral CT scan of thoracic spine demonstrates T3/4 fracture dislocation (white arrow).

The patient tolerated the diet well and these measures led to a dramatic decrease in the chest tube output to less than $100 \mathrm{ml} /$ day of serous fluid by the time he had operative repair and stabilization of his thoracic spine on hospital day seven. After the surgical procedure there was a transient increase in output from the chest tube to $200 \mathrm{ml}$ per day which declined to $35 \mathrm{ml}$ on hospital day 14 . The chest tube was then removed without consequence, he was then started on a regular diet and follow up chest $\mathrm{x}$-rays did not reveal any recurrent pleural effusions. The patient was discharged to an inpatient rehabilitation facility and was seen approximately two months after his injury in our clinic. He still had complete motor paralysis of the lower extremities with a T2 sensory loss. His upper extremity function remained unchanged from admission with his motor function intact. His pulmonary status remained stable as he had no ongoing acute pulmonary issues and saturated $98-100 \%$ on room air.

\section{Discussion}

Chylous pleural effusion is most commonly caused by malignancy, accounting for more than $50 \%$ of cases. Lymphoma is the most common malignant cause, representing about $60 \%$ of all cases, with the non-Hodgkins variant being the most prevalent. Traumatic injuries to the upper abdomen and chest including those sustained 
during surgery are the second leading cause of chylothorax, accounting for approximately $25 \%$ of cases.

The first traumatic injury to the thoracic duct was described in 1875 and the first thoracic duct ligation was performed in 1948 [6]. The traumatic causes of injury to the duct vary widely, and the most common blunt mechanism producing injury is related to sudden hyperextension of the spine with rupture of the duct just above the diaphragm [4,7-9]. Sudden stretching over the vertebral bodies for any reason may tear the duct, but this usually occurs in the setting of a thoracic duct previously affected by disease $[4,8]$. Episodes of vomiting or a violent bout of coughing resulting in shearing of the lymphatic conduit along the crux of the right diaphragm has been reported as well [9]. Penetrating injuries, from a gunshot or stab wound, are less common and usually associated with severe damage to nearby structures.

The pertinent anatomy involved in the development of a chylothorax begins with the cysterna chyli, which is a confluence of lymphatics located in the retroperitoneum, just to the right of the posteromedial aorta at the level of the renal arteries. The thoracic duct ascends from this level and enters the chest through the aortic hiatus into the right hemithorax. The duct crosses over to the left chest at the fourth and fifth thoracic levels and enters the neck anterior to the left subclavian artery to join the venous system at the junction of the left subclavian vein and left internal jugular vein $[10,11,13]$. Knowledge of this anatomy should alert the physician to the possibility of a thoracic duct injury with thoracic spine fractures or any associated upper abdomen or chest injury involving this trajectory.

As in this case, the diagnosis of a chyle leak was supported by a pleural fluid triglyceride level greater than $110 \mathrm{mg} / \mathrm{dL}$. A pleural fluid triglyceride concentration less than $50 \mathrm{mg} / \mathrm{dL}$ excludes a chylothorax. An intermediate level between 50 and $110 \mathrm{mg} / \mathrm{dL}$ should be followed by lipoprotein analysis to inspect the pleural fluid for chylomicrons or cholesterol crystals. The presence of chylomicrons and the absence of cholesterol crystals confirm a chyle leak. In addition, a ratio of pleural fluid cholesterol to triglyceride of less than 1 is also diagnostic $[11,12]$.

Although most cases of traumatic chylothorax can be managed non-operatively, the need for surgical intervention in the subset of patients with associated thoracic fractures is higher and approaches 50 percent $[5,11]$. Indications for surgical intervention include an excessive leak for greater than five days, which for adults is $>500 \mathrm{ml} /$ day and for children $>10 \mathrm{ml} / \mathrm{kg} /$ day or $>100 \mathrm{ml} /$ day for each year of age. Any volume of output for greater than 14 days would indicate failure of conservative therapy and the need for surgical intervention as well [10].

Conservative treatment of chylothorax begins with prompt drainage via tube thoracostomy and dietary manipulation. The goal of dietary manipulation is to dramatically decrease lymph production since sixty percent of the dietary fat is absorbed by the lymphatic system, and approximately 1500 to $2000 \mathrm{ml}$ of lymph is produced daily. This can be accomplished by completely bypassing the lymphatic circulation with Total Parenteral Nutrition (TPN), or by strict dietary manipulation based on a very low fat diet. We chose the latter to avoid some of the known infectious complications associated with TPN, especially in this high risk patient with multi-system trauma. With either approach, volume status, electrolyte abnormalities and nutritional parameters of the patient should be followed closely and aggressive replacement of nutritional losses of fat soluble vitamins and proteins should be carried out $[10,14,15]$.

A diet strategy to limit chyle production involves avoidance of long chain triglycerides (LCTs). Medium chain triglycerides (MCTs), however, are absorbed directly into the portal circulation without stimulating lymphatic flow, so inclusion of these MCTs in the diet can help to increase caloric intake for healing [11]. These dietary changes as in our case were accomplished by a strict low fat diet supplemented with extra protein powder and MCT oil. Elemental peptide-based enteric formulas with less than 3\% LCTs and MCTs added are also ideal supplements, as they have been shown to reduce the quantity and duration of chyle output. Though there is no consensus on the definitive duration of this nutritional management, the literature supports that these dietary measures be continued for approximately two weeks [10-12]. In general, once the chyle leak is resolved, then a regular diet may be resumed. In complicated cases, however, it may be advisable to ensure leak resolution with a provocative high-fat meal before removing a drainage tube.

Finally, there are anecdotal data supporting the use of octreotide to promote decreased lymphatic production. The mechanism is based on the reduction of gastrointestinal secretions and sphlancnic blood flow associated with octreotide, thus decreasing lymphatic production. The drug can be given as a continuous infusion or bolus injections [16,17].

\section{Conclusions}

Although rare, traumatic chylothorax can be a difficult entity to manage especially in a patient with additional traumatic injuries. This case reflects a successful approach to the management of a traumatic chyle leak, using drainage and strict dietary changes, which precluded the need for surgical intervention.

\section{Consent}

Written informed consent was obtained from the patient for publication of this case report and accompanying 
images. A copy of the written consent is available for review by the Editor-in-Chief of this journal.

\section{Author details}

'Department of Surgery, Division of Trauma and Surgical Critical Care, Kern Medical Center, Bakersfield, California. ${ }^{2}$ Department of Nutrition and Dietary Services, Kern Medical Center, Bakersfield, California.

\section{Authors' contributions}

All authors contributed to researching, editing and writing the article. All authors read and approved the final manuscript.

\section{Competing interests}

The authors declare that they have no competing interests.

Received: 8 January 2011 Accepted: 28 March 2011

Published: 28 March 2011

\section{References}

1. Maldonado F: Medical and surgical management of chylothorax and associated outcomes. Am J Med Sci 2010, 339(4):314-318.

2. Doerr CH, Allen MS, Nichols FC, Ryu JH: Etiology of Chylothorax in 203 patients. Mayo Clinic Proc 2005, 80(7):867-870.

3. Fogli L, Gorini P, Belcastro S: Conservative management of traumatic chylothorax: a case report. Intens Care Med 1993, 19(3):176-177.

4. Valentine V, Raffin T: The management of chylothorax. Chest 1992, 102:586-591.

5. Paul S: Surgical management of chylothorax. Thorac Cardiovasc Surg 2009, 57(4):226-228.

6. Browse NL, Allen DR, Wilson NM: Management of chylothorax. Br J Surg 1997, 84(12):1711-1716.

7. Wright $P$, Gardner A: Traumatic chylothorax: A case after dislocation of the thoracic spine. J Bone Joint Surg 1952, 34B:64-67.

8. Brook M, Dupree D: Bilateral traumatic chylothorax. Bilateral traumatic chylothorax 1988, 17:69-72.

9. Biet $A B$, Connolly NK: Traumatic chylothorax; a report of a case and a survey of literature. Br J Surg 1951, 39:564-568.

10. Spain DA: The A.S.P.E.N. Nutrition Support Core Curriculum: A CaseBased Approach-The Adult Patient. American Society of Parenteral and Enteral Nutrition 2007, 477-487.

11. Mason JF: Chylothorax. In Murray and Nadel's Textbook of Respiratory Medicine. 5 edition. Edited by: Murray JF, Nadel JA. Philadelphia: Saunders; 2010:1764-1792.

12. Staats BA, Ellefson RD, Budahn LL, Dines DE, Prakash UB, Offord UK: The lipoprotein profile of chylous and nonchylous pleural effusions. Mayo Clin Proc 1980, 55:700-704.

13. Miller JJ: Anatomy of the thoracic duct \& chylothorax. In General Thoracic Surgery. 6 edition. Edited by: Shields T, Loccicero J, Ponn R, et al. Philadelphia: Lippincott, Williams 2005:879-888.

14. Sahn SA: Pleural effusions of extra vascular origin. Clin of Chest Med 2006, 285-308.

15. Apostolakis E: Traumatic chylothorax following blunt trauma: two conservatively treated cases. J Card Surg 2009, 24(2):220-2.

16. Barili F: Administration of octreotide for management of postoperative high-flow chylothorax. Ann Vasc Surg 2007, 21(1):90-92.

17. Miura K: Treatment of a persistent postoperative chylothorax with octreotide. Kyobu Geka 2009, 62(10):885-887.

doi:10.1186/1749-7922-6-10

Cite this article as: Pakula et al: A case of a traumatic chyle leak following an acute thoracic spine injury: successful resolution with strict dietary manipulation. World Journal of Emergency Surgery 2011 6:10.

\section{Submit your next manuscript to BioMed Central and take full advantage of:}

- Convenient online submission

- Thorough peer review

- No space constraints or color figure charges

- Immediate publication on acceptance

- Inclusion in PubMed, CAS, Scopus and Google Scholar

- Research which is freely available for redistribution

Submit your manuscript at www.biomedcentral.com/submit
Ciomed Central 